\title{
Effect of Exercise with Rhythmic Auditory Stimulation on Muscle Coordination and Gait Stability in Patients with Diabetic Peripheral Neuropathy: A Randomized Controlled Trial
}

\author{
Keisuke Suzuki ${ }^{*}$, Masaya Niitsu ${ }^{2}$, Tomohiko Kamo ${ }^{3}$, Satoshi Otake1, Yusuke Nishida4 \\ ${ }^{1}$ Department of Physical Therapy, International University of Health and Welfare, Odawara, Japan \\ ${ }^{2}$ Department of Rehabilitation, Iwata City Hospital, Iwata, Japan \\ ${ }^{3}$ Department of Physical Therapy, Japan University of Health Sciences, Satte, Japan \\ ${ }^{4}$ Department of Physical Therapy, International University of Health and Welfare, Narita, Japan \\ Email ^keisukedondon@gmail.com
}

How to cite this paper: Suzuki, K., Niitsu, M., Kamo, T., Otake, S. and Nishida, Y. (2019) Effect of Exercise with Rhythmic Auditory Stimulation on Muscle Coordination and Gait Stability in Patients with Diabetic Peripheral Neuropathy: A Randomized Controlled Trial. Open Journal of Therapy and Rehabilitation, 7, 79-91. https://doi.org/10.4236/ojtr.2019.73005

Received: May 16, 2019

Accepted: June 2, 2019

Published: June 5, 2019

Copyright $\odot 2019$ by author(s) and Scientific Research Publishing Inc. This work is licensed under the Creative Commons Attribution International License (CC BY 4.0).

http://creativecommons.org/licenses/by/4.0/

\begin{abstract}
Background: Diabetic peripheral neuropathy (DPN) changes leg muscle coordination during walking and reduces stability. The aim of this study was to determine whether rhythmic auditory stimulation (RAS) affected the gait performance of patients with DPN. Methods: Forty DPN patients (mean age, $59.1 \pm 9.4$ y) were randomly allocated to RAS and control groups in equal numbers. The participants in each group underwent 2 weeks of supervised rehabilitative treatment $(40 \mathrm{~min} /$ day $)$ as inpatients. This included walking twice a day, during which the RAS group participants walked in time with a metronome set at a self-chosen, comfortable rate. We compared gait function, lower limb muscle co-contraction, and gait stability before and after the intervention for both groups, calculated the change in score for each parameter, and assessed differences between the groups with unpaired t-tests and ANCOVA. Results: RAS was associated with significant improvement in all parameters. In the control group, there was no improvement in cadence, co-contraction, or gait stability (vertical). Compared with the control group, the RAS group showed improvement in co-contraction and gait stability. Conclusion: RAS may be helpful for improving the lower limb muscle coordination and gait function of DPN patients.
\end{abstract}

\section{Keywords}

Co-Contraction, Root Mean Square, Muscle Activity, Gait Stability, Auditory Cue 


\section{Introduction}

Diabetic peripheral neuropathy (DPN) afflicts more than half of all diabetic patients and is associated with a particularly high level of morbidity [1]. DPN patients with diminished plantar sensation have been observed to exhibit increased postural sway along with a significant loss of postural control [2]. This difficulty in postural control coupled with an altered gait pattern increases their risk of falls [3].

DPN impairs both the somatosensory and the motor control system, affecting the amount and quality of sensory information gained, information that is essential for the complex generation and control of gait. This results in greater instability during gait and static posture [4], which previously was ascribed to muscular weakness. Gait in humans is considered to result from coordinated interplay between neural and muscular actions in coordination with the skeletal function [5]. Changes in this relationship due to the loss of sensitivity can result in important biomechanical alteration to the patient's gait. These include changes in plantar pressure, usually with increased pressure on the forefoot [6], differences in kinetic patterns with modified ground reaction forces [7], and altered muscle activity [8]. Compared with healthy controls, DPN patients have a lower gait velocity, decreased cadence, shorter stride length, increased stance time, and greater step-to-step variability [9]. These gait alterations increase when walking on irregular surfaces [9]. DPN patients also exhibit lower ankle moment and ankle power compared with healthy controls, as well as a different onset and cessation time of muscle activity [5]. These patients present more co-contractions of agonist and antagonist muscles at the ankle and knee joints during the stance phase. Kwon et al. [5] speculated that the co-contraction mechanism may enable these individuals to adopt a safer, more stable gait pattern to compensate for their diminished sensory information.

General exercises for gait and balance improvement have previously been reported to be effective for DPN patients. Allet et al. [10] reported a randomized controlled trial in which a group that received specific training, which consisted of circuit training gait and balance exercises over 12 weeks in a real-life environment, showed a significant improvement in gait parameters. These results demonstrated that it is possible to improve functional and independent gait, even with sensory and motor impairments [11]. Ahn et al. [12] showed a significant improvement in balance and neuropathic symptoms in a group that received a standardized Tai Chi for diabetes program, which comprised a 1-hour Tai Chi session twice a week for 12 weeks. However, DPN patients have a dropout rate during interventions. For example, the dropout rate for Ahn et al. [12] 12-week Tai Chi intervention exceeded $30 \%$. It is therefore necessary to establish a way to treat lower limb function and gait in DPN patients that minimizes dropout.

Several studies have used rhythmic auditory stimulation (RAS) to help improvement of gait [13] [14] [15] [16]. The influence of rhythmic sensory cues on 
walking dynamics is of considerable relevance to neurological rehabilitation. In this regard, RAS has shown positive effects on various gait characteristics for patients with Parkinson's disease [13] and hemiparesis [14]. Passively listening to rhythmic stimuli, even in the absence of motor actions or intent, recruits the auditory systems as well as the mid-premotor cortex and the supplementary motor area [17]. Through a process known as rhythmic entrainment, people naturally move in synchrony to external rhythmic cues [18]. In addition, Ashoori, et al. [13] found that auditory stimuli reduced the amount of time required for muscles in healthy human subjects to respond to a given motor command, compared to the absence of stimuli; the authors explained this as RAS patterns increasing the excitability of spinal motor neurons. Thus, RAS can influence conscious and subconscious abilities and thereby improve gait function [15]. Furthermore, this improvement has been demonstrated with short interventions lasting a matter of several weeks in stroke and Parkinson's disease patients [16].

However, little is known about the effect of RAS on the gait of DPN patients. We hypothesized that intervention using RAS would improve their muscle activity and gait function over a short period. The purpose of this study was to determine whether intervention using RAS affected the lower limb muscle coordination and gait performance of patients with DPN.

\section{Methods}

\subsection{Participants}

This study was conducted from August 2013 to April 2015. The participants were adult patients with type 2 diabetes mellitus with DPN recruited from the Endocrinology Department of Iwata City Hospital. Exclusion criteria included pregnancy, taking corticosteroids, hormone replacement therapy, osteoporosis, chronic renal failure, chronic heart failure, current bilateral foot ulceration, severe retinopathy, neuropathic pain, malignancy, and endocrine disorders other than diabetes. The inclusion criteria were the ability to walk independently without assistance, hearing capacity sufficient to perceive the auditory cues, and the absence of significant cognitive impairment (such as a Mini-Mental Status Examination score $>24$ ) (Figure 1).

Prospective participants were informed that if they participated they would be randomly assigned to an intervention group or a control group. Those who agreed to join the study were contacted by the study coordinator for an individual appointment, at which the informed consent document was signed and a baseline evaluation performed. The study conformed to the principles of the Declaration of Helsinki, and informed consent was obtained from all participants. The protocol was approved by the Ethics Committee of the International University of Health and Welfare. This study was registered as a clinical trial (UMIN000026345).

Forty participants took part in the study (mean \pm standard deviation (SD) age, $59.1 \pm 9.4 \mathrm{y}$; weight, $69.4 \pm 20.3 \mathrm{~kg}$; height, $1.61 \pm 0.09 \mathrm{~m}$; and body mass index 


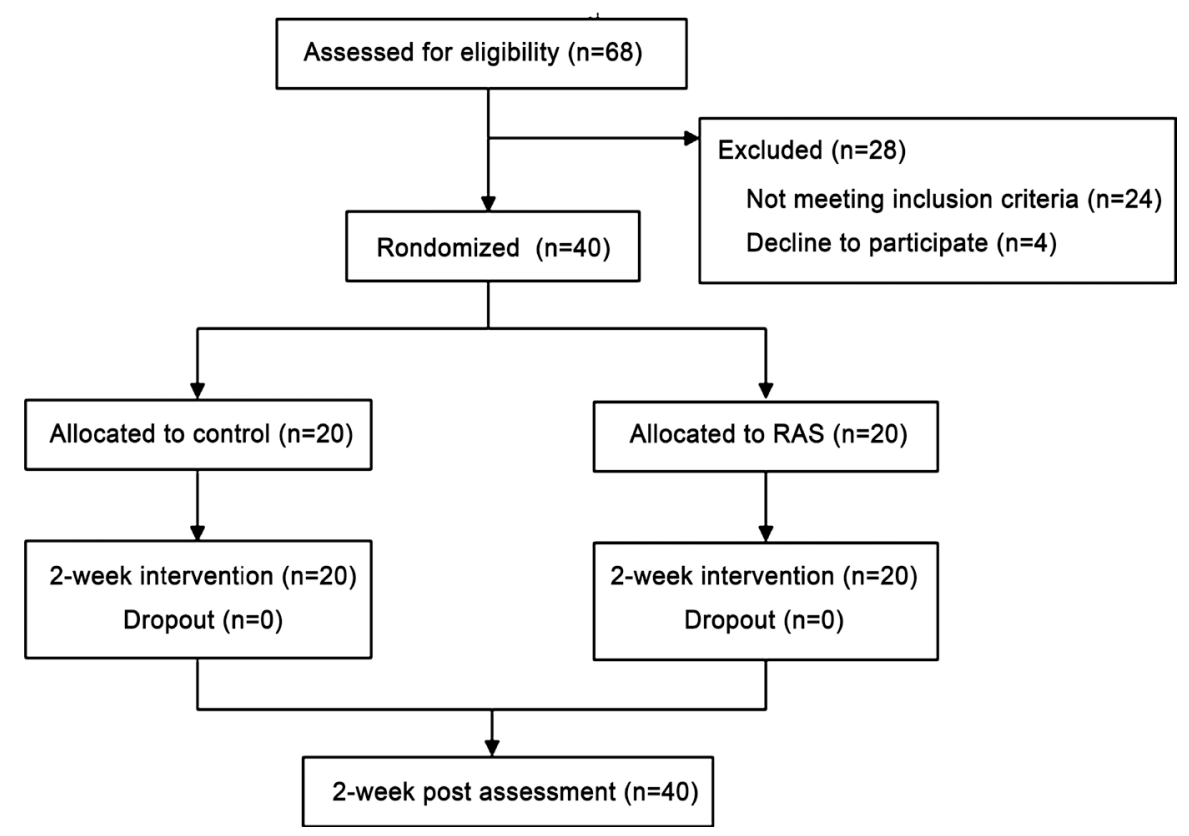

Figure 1. Study design schematic.

(BMI), $26.3 \pm 5.7 \mathrm{~kg} / \mathrm{m}^{2}$ ). The participants were divided into two groups: 20 were allocated to the RAS intervention group and 20 to the control group. There were no significant differences between the two groups in terms of their general characteristics.

\subsection{The Rehabilitation Protocol}

The participants in each group performed 2 weeks of supervised rehabilitative treatment $(40 \mathrm{~min} /$ day) as inpatients, assisted in their training by a certified physical therapist. The typical training session included a set of exercises aimed to muscle strength, balance, and physical endurance, as well as specific gait training [10]. In particular, 20 min of each session was dedicated to continuous level walking, during which the RAS group participants listened to auditory cues given by a portable electronic metronome (ME-55, Yamaha, Japan). During the 2-week period, the RAS group participants were also instructed to perform at least two further unsupervised 20-min training sessions each day after meals, including gait training with RAS. All participants were provided with a diary in which they self-reported the duration and types of activities they performed. The diary was reviewed by the physical therapists five times a week.

The RAS consisted of auditory beats whose frequency (bpm) was personalized for each participant at the initial gait assessment performed at the beginning of the study, setting a gait speed comfortable for the participant. The pace that reflects on imposed gait cadence during the training was set on the basis of the difference between the cadences of each participant. The participants were allowed to change the tempo of the metronome beats themselves.

The control group did the same amount of walking as the RAS group, albeit without the metronome. 


\subsection{Sensory Neuropathy Assessment}

The diagnosis of diabetic neuropathy followed the recommendations of the simplified diagnostic criteria proposed by the Diabetic Neuropathy Study Group in Japan [19], and was based on the presence of two of the following three factors: 1) subjective symptoms in the bilateral lower limbs or feet;2) absent or reduced ankle jerk; and 3) decreased vibration perception, assessed using a C128 tuning fork and measured bilaterally at the medial malleoli.

\subsection{Step Count}

For the purposes of this study, "number of steps" refers specifically to the average daily step count as recorded by a pedometer (PD-635, TANITA, Japan). Participants were given a pedometer and were instructed to wear the monitor around their waist during all waking hours. The participants were given the monitor for at least 14 consecutive days, and the average daily step count was calculated. For a day to be included, the monitor had to be worn for at least 10 hours. During the intervention period, the participants recorded the pedometer data in their exercise diaries.

\subsection{Assessment of Gait Parameters}

All of the participants wore their usual shoes, although low-heeled footwear such as sandals or slippers were excluded. The participants were asked to walk, at their own preferred speed, along a $10-\mathrm{m}$ path in a corridor with a flat floor with no inclination. The gait speed was calculated as $10 \mathrm{~m}$ divided by the walking time in seconds. The step length was calculated from the number of steps taken over the $10 \mathrm{~m}$. The cadence was then calculated from the $10-\mathrm{m}$ gait time and number of steps.

\subsection{Gait Assessment: $E M G$}

Electromyographic (EMG) activity was recorded for two muscles, the tibialis anterior (TA) and soleus (SOL), in the participant's right leg using active electrodes (BlueSensor M-00-S, Ambu, Denmark) applied to lightly abraded skin over the respective muscle belly. Electrode placement was carefully chosen to minimize cross-talk from adjacent muscles. The signals were amplified, filtered to 20 - 450 $\mathrm{Hz}$ (Tele Myo G2, EM-601, NORAXON Inc., USA), sampled at $1500 \mathrm{~Hz}$, and recorded onto disk. The gait cycle was defined by consecutive foot contacts, as determined by signals from a footswitch (EM434, NORAXON Inc., USA). The $E M G$ data were rectified and then interpolated to 100 evenly spaced values for each instance the foot triggered the footswitch. $E M G$ datum traces were then normalized by their largest value for each step. The mean of the linear envelope of the $E M G$ signal was obtained $100 \mathrm{~ms}$ before and after the initial contacts of the first ten strides. All the linear envelope values were normalized to the peak activation during the gait [20]. To measure the antagonist co-contraction, we calculated the ratio of activation between the shank muscles (TA/SOL). The 
co-contraction index $(C I)$ was calculated using the following mathematical equation, adapted from Falconer et al. [21]:

$$
\begin{gathered}
I_{\text {ant }}=\int_{t 1}^{t 2} E M G_{T A}(t) \mathrm{d} t+\int_{t 2}^{t 3} E M G_{S O L}(t) \mathrm{d} t \\
I_{\text {total }}=\int_{t 1}^{t 3}\left[E M G_{\text {agon }}+E M G_{\text {ant }}\right](t) \mathrm{d} t \\
C I=\frac{2 I_{\text {ant }}}{I_{\text {tortal }}} \times 100 \%
\end{gathered}
$$

where $I_{\text {ant }}$ is the area of the total antagonistic activity. where $t 1$ to $t 2$ denotes the period during which the TA $E M G$ is less than the SOL $E M G$ and $t 2$ to $t 3$ donates the period during which the SOL EMG is less than the TA EMG), Itotal is the integral of the sum of TA and SOL EMG. Because we were comparing assessments before and after the intervention, we placed a marker as a substitute for the electrodes to ensure we assessed the same muscle activity.

\subsection{Gait Assessment: Triaxial Accelerometer}

A triaxial accelerometer (WAA-001, Wireless Technologies Inc., Japan) was clipped tightly onto a band placed securely around the participant's waist such that it was located approximately at the center of the spine at the L3 level, in order to measure the acceleration of the back and waist in the mediolateral $(M L)$, vertical $(V)$, and anteroposterior $(A P)$ directions [22]. This position was chosen owing to its proximity to the center of mass of the human body when standing. The signals were sampled at $200 \mathrm{~Hz}$. After corrections of the signal, the root mean square $(R M S)$ values for each direction were calculated using the following equations:

$$
\begin{gathered}
R M S_{X}=\frac{\sqrt{\frac{1}{N} \sum_{i=1}^{N}\left(x_{i}\right)^{2}}}{v^{2}} \\
R M S_{T}=R M S_{M L}+R M S_{V}+R M S_{A P}
\end{gathered}
$$

where $X$ represents the direction of acceleration $(M L, V$, or $A P)$ and $T$ is the total. The $R M S$ is a statistical measure of the magnitude of acceleration in each direction. Menz et al. [23] reported that the $R M S$ demonstrated a non-linear relationship to walking speed. The $R M S$ values were therefore normalized by the square of the walking speed.

\subsection{Statistical Analyses}

To evaluate the effects of the interventions, we used paired t-tests to compare parameter values before and after each intervention. We calculated the change in each parameter (post-training minus pre-training) and compared these between the two groups using unpaired t-tests and ANCOVA with the change in score as the dependent variable and the treatment group (RAS or control group) as the independent variable. Age, sex, BMI, years of DM, and activity during the intervention (steps/day) categories were entered as the covariates. We also calculated 
the effect size (Cohen's d) for each statistic by dividing the pre-post change in the parameter by its pooled SD [24]. An effect size of $d=0.2$ was considered as indicating a small effect, 0.5 as medium, and 0.8 or above as large. A p-value < 0.05 was considered statistically significant.

\section{Results}

Table 1 summarizes the characteristics of the participants. The mean and standard deviation of age was $59 \pm 9$ years. Body mass index was $26.32 \pm 5.69 \mathrm{~kg} / \mathrm{m}^{2}$. The average of Vibration perception was relatively low $(7.86 \pm 3.04 \mathrm{sec})$ suggesting poor sensory function in our sample. Subjective symptoms were reported in $15 \%$ of participants, while absent or reduced ankle jerk was reported in $73 \%$

Table 1. Descriptive characteristics of the participants.

\begin{tabular}{|c|c|c|}
\hline & $\operatorname{RAS}(\mathrm{n}=20)$ & Control $(\mathrm{n}=20)$ \\
\hline Age (years) & $59 \pm 6$ & $59 \pm 11$ \\
\hline Sex (male) & $10(50 \%)$ & $13(65 \%)$ \\
\hline Height $(\mathrm{cm})$ & $161.94 \pm 9.41$ & $161.29 \pm 7.78$ \\
\hline Weight $(\mathrm{kg})$ & $69.46 \pm 25.61$ & $69.41 \pm 13.90$ \\
\hline BMI $\left(\mathrm{kg} / \mathrm{m}^{2}\right)$ & $26.09 \pm 6.98$ & $26.55 \pm 4.20$ \\
\hline Duration of DM (years) & $5.8 \pm 8.1$ & $6.9 \pm 8.5$ \\
\hline Vibration perception (s) & $7.42 \pm 3.03$ & $8.29 \pm 3.05$ \\
\hline Subjective symptoms (n) & $3(15 \%)$ & $3(15 \%)$ \\
\hline Absent or reduced ankle jerk (n) & $15(75 \%)$ & $14(70 \%)$ \\
\hline Hypertension (n) & $10(50 \%)$ & $11(55 \%)$ \\
\hline Hyperlipidemia (n) & $14(70 \%)$ & $15(75 \%)$ \\
\hline Drinking history (n) & $12(60 \%)$ & $10(50 \%)$ \\
\hline Smoking history (n) & $8(40 \%)$ & $11(55 \%)$ \\
\hline Diabetic renal disease (>stage III) & $2(10 \%)$ & $4(20 \%)$ \\
\hline Diabetic retinopathy (>PPDR) & $1(5 \%)$ & $0(0 \%)$ \\
\hline Insulin treatment & $3(15 \%)$ & $1(5 \%)$ \\
\hline HbAlc (\%) & $11.52 \pm 2.15$ & $10.89 \pm 2.02$ \\
\hline Plasma glucose (mg/dl) & $317.65 \pm 117.5$ & $277.65 \pm 124.74$ \\
\hline LDL-C (mg/dl) & $129.00 \pm 31.40$ & $121.10 \pm 42.97$ \\
\hline $\operatorname{ALT}(\mathrm{IU} / \mathrm{L})$ & $36.9 \pm 25.10$ & $44.25 \pm 35.11$ \\
\hline$\gamma$-GPT (IU/L) & $105.80 \pm 250.22$ & $55.50 \pm 44.87$ \\
\hline $\mathrm{TG}(\mathrm{mg} / \mathrm{dl})$ & $200.16 \pm 190.32$ & $177.95 \pm 136.13$ \\
\hline Steps during intervention (steps/day) & $7506.01 \pm 2090.92$ & $6524.96 \pm 1933.91$ \\
\hline
\end{tabular}

Values are mean \pm standard deviation or number (\%). RAS, rhythmic auditory stimulation group; BMI, body mass index; DM, diabetes mellitus; PPDR, preproliferative diabetic retinopathy; LDL-C, low-density lipoprotein cholesterol; ALT, alanine aminotransferase; $\gamma$-GPT, gamma-glutamyl transpeptidase; TG, triglyceride. $\mathrm{p}<0.05$ (unpaired $\mathrm{t}$ test, $\chi^{2}$ test) RAS vs Control groups. 
of participants. Almost all participants were hypertension (53\%) or Hyperlipidemia (73\%). Diabetic renal disease (>stage III) was reported for $15 \%$ of participants. Few were current diabetic retinopathy (>PPDR). The average of HbA1c was relatively high $(11.21 \% \pm 2.07 \%)$. There were no significant differences between the two groups for any of these parameters.

Table 2 presents a comparison of the gait parameters and $R M S$ waist acceleration values before and after the intervention in the two groups. After the intervention, the RAS group showed significant increases in gait speed, step length, and cadence, and decreases in $C I, R M S_{M L}, R M S_{V}, R M S_{A P}$ and $R M S_{T}(\mathrm{p}<0.05)$. The control group showed significant increases in gait speed and step length, and decreases in $R M S_{M L}, R M S_{A P}, R M S_{T}$ ( $\left.\mathrm{p}<0.05\right)$; however, no statistically significant changes were observed for cadence $(\mathrm{p}=0.29), C I(\mathrm{p}=0.42)$, or $R M S_{V}(\mathrm{p}$ $=0.19$ ).

Table 3 compares the changes in gait and $R M S$ parameters between the RAS and control groups. The reduction in $C I$ was significantly greater in the RAS group than in the control group $(\mathrm{p}<0.05)$. Similarly, the RAS group showed significantly greater reductions than the control group in $R M S_{M L}$ and $R M S_{T}(\mathrm{p}<$ $0.05)$. No significant differences between the groups were observed for the changes in gait speed $(\mathrm{p}=0.20)$, step length $(\mathrm{p}=0.81)$, cadence $(\mathrm{p}=0.15), R M S_{V}$ ( $\mathrm{p}=0.13)$, or $R M S_{A P}(\mathrm{p}=0.16)$.

Table 4 presents a comparison between the RAS and control groups of the changes in parameters adjusted for the covariates. ANCOVA analysis showed that $C I$ and $R M S_{T}$ were reduced to a significantly greater extent in the RAS group than in the control group, but that changes in other gait parameters did not differ significantly between the groups.

Table 2. The measured gait parameters and $R M S$ waist acceleration values before and after the intervention in the rhythmic auditory stimulation (RAS) and control groups.

\begin{tabular}{ccccccc}
\hline & \multicolumn{3}{c}{ RAS } & \multicolumn{2}{c}{ Control } & \\
\cline { 2 - 7 } & $\begin{array}{c}\text { Before } \\
\text { intervention }\end{array}$ & $\begin{array}{c}\text { After } \\
\text { intervention }\end{array}$ & $\begin{array}{c}\text { Cohen's } \\
\mathrm{d}\end{array}$ & $\begin{array}{c}\text { Before } \\
\text { intervention }\end{array}$ & $\begin{array}{c}\text { After } \\
\text { intervention }\end{array}$ & $\begin{array}{c}\text { Cohen's } \\
\mathrm{d}\end{array}$ \\
\hline Gait speed (m/s) & $1.29 \pm 0.19$ & $1.50 \pm 0.19^{*}$ & 1.10 & $1.25 \pm 0.19$ & $1.38 \pm 0.22^{*}$ & 0.65 \\
Step length (m) & $0.62 \pm 0.07$ & $0.67 \pm 0.06^{*}$ & 0.81 & $0.60 \pm 0.09$ & $0.64 \pm 0.08^{*}$ & 0.51 \\
Cadence & $124.25 \pm 11.16$ & $131.07 \pm 9.06^{*}$ & 0.67 & $122.93 \pm 7.43$ & $125.26 \pm 10.74$ & 0.25 \\
$($ step/min) & & & & & & \\
$C I(\%)$ & $49.31 \pm 11.82$ & $42.70 \pm 11.55^{*}$ & 0.57 & $48.31 \pm 11.43$ & $47.09 \pm 10.05$ & 0.11 \\
$R M S_{M L}\left(\mathrm{~m} / \mathrm{s}^{2}\right)$ & $0.53 \pm 0.16$ & $0.41 \pm 0.11^{*}$ & 0.93 & $0.58 \pm 0.31$ & $0.53 \pm 0.31^{*}$ & 0.63 \\
$R M S_{V}\left(\mathrm{~m} / \mathrm{s}^{2}\right)$ & $1.42 \pm 0.28$ & $1.28 \pm 0.25^{*}$ & 0.52 & $1.43 \pm 0.23$ & $1.37 \pm 0.23$ & 0.25 \\
$R M S_{A P}\left(\mathrm{~m} / \mathrm{s}^{2}\right)$ & $0.83 \pm 0.19$ & $0.69 \pm 0.13^{*}$ & 0.85 & $0.85 \pm 0.17$ & $0.78 \pm 0.13^{*}$ & 0.47 \\
$R M S_{T}\left(\mathrm{~m} / \mathrm{s}^{2}\right)$ & $2.78 \pm 0.50$ & $2.38 \pm 0.40^{*}$ & 0.87 & $2.86 \pm 0.52$ & $2.68 \pm 0.52^{*}$ & 0.37 \\
\hline
\end{tabular}

Values are mean \pm standard deviation. $C I$, co-contraction index; $R M S$, root mean square $(M L$, mediolateral; $V$, vertical; $A P$, anteroposterior; $\mathrm{T}$, total). ${ }^{*} \mathrm{p}<0.05$ (paired t test), before intervention vs after intervention. Effect size, Cohen's d: $<0.20$, no effect; $0.21-0.50$, a small effect; $0.51-0.80$, a medium effect; and $>0.80$, a large effect. 
Table 3. Comparison of changes $(\Delta)$ in gait parameters and $R M S$ waist acceleration values between the rhythmic auditory stimulation (RAS) and control groups.

\begin{tabular}{cccc}
\hline & RAS & Control & Cohen's d \\
\hline$\Delta$ Gait speed $(\mathrm{m} / \mathrm{s})$ & $0.21 \pm 0.17$ & $0.13 \pm 0.16$ & 0.79 \\
$\Delta$ Step length $(\mathrm{m})$ & $0.05 \pm 0.07$ & $0.04 \pm 0.05$ & 0.08 \\
$\Delta$ Cadence (step/min) & $6.81 \pm 9.77$ & $2.33 \pm 9.6$ & 0.46 \\
$\Delta C I(\%)$ & $-6.61 \pm 7.81^{*}$ & $-0.89 \pm 6.70$ & 0.44 \\
$\Delta R M S_{M L}\left(\mathrm{~m} / \mathrm{s}^{2}\right)$ & $-0.12 \pm 0.09^{*}$ & $-0.05 \pm 0.11$ & 0.69 \\
$\Delta R M S_{V}\left(\mathrm{~m} / \mathrm{s}^{2}\right)$ & $-0.14 \pm 0.15$ & $-0.05 \pm 0.18$ & 0.50 \\
$\Delta R M S_{A P}\left(\mathrm{~m} / \mathrm{s}^{2}\right)$ & $-0.14 \pm 0.14$ & $-0.07 \pm 0.14$ & 0.45 \\
$\Delta R M S_{T}\left(\mathrm{~m} / \mathrm{s}^{2}\right)$ & $-0.40 \pm 0.31^{*}$ & $-0.18 \pm 0.37$ & 0.65 \\
\hline
\end{tabular}

Values are mean \pm standard deviation. $C I$, co-contraction index; $R M S$, root mean square ( $M L$, mediolateral; $V$, vertical; $A P$, anteroposterior; $\mathrm{T}$, total). ${ }^{*} \mathrm{p}<0.05$ (unpaired t test) $\triangle \mathrm{RAS}$ vs $\Delta$ Control. Effect size, Cohen's $\mathrm{d}:<0.20$, no effect; $0.21-0.50$, a small effect; $0.51-0.80$, a medium effect; and $>0.80$, a large effect.

Table 4. Comparison of the adjusted changes $(\Delta)$ in gait parameters and $R M S$ waist acceleration values between the rhythmic auditory stimulation (RAS) and control groups.

\begin{tabular}{ccc}
\hline & RAS & Control \\
\hline$\Delta$ Gait speed $(\mathrm{m} / \mathrm{s})$ & $0.19 \pm 0.04$ & $0.15 \pm 0.04$ \\
$\Delta$ Step length $(\mathrm{m})$ & $0.05 \pm 0.01$ & $0.04 \pm 0.01$ \\
$\Delta$ Cadence $($ step $/ \mathrm{min})$ & $6.35 \pm 2.31$ & $2.79 \pm 2.31$ \\
$\Delta C I(\%)$ & $-6.91 \pm 1.75^{*}$ & $-0.58 \pm 1.75$ \\
$\Delta R M S_{M L}\left(\mathrm{~m} / \mathrm{s}^{2}\right)$ & $-0.12 \pm 0.02$ & $-0.06 \pm 0.02$ \\
$\Delta R M S_{V}\left(\mathrm{~m} / \mathrm{s}^{2}\right)$ & $-0.15 \pm 0.04$ & $-0.04 \pm 0.04$ \\
$\Delta R M S_{A P}\left(\mathrm{~m} / \mathrm{s}^{2}\right)$ & $-0.15 \pm 0.03$ & $-0.06 \pm 0.03$ \\
$\Delta R M S_{T}\left(\mathrm{~m} / \mathrm{s}^{2}\right)$ & $-0.42 \pm 0.08^{*}$ & $-0.16 \pm 0.08$ \\
\hline
\end{tabular}

Values are mean \pm standard error. $C I$, co-contraction index; $R M S$, root mean square $(M L$, mediolateral; $V$, vertical; $A P$, anteroposterior; $\mathrm{T}$, total). ${ }^{*} \mathrm{p}<0.05$ (ANCOVA) $\triangle \mathrm{RAS}$ vs $\triangle \mathrm{Control}$. The covariates were age, sex, body mass index, duration of diabetes (y), and activity during the intervention (steps per day) categories.

\section{Discussion}

The main aim of the present study was to assess the effectiveness of 2 weeks of treatment that included gait training assisted by RAS. All of the participants completed the program. Our results showed that RAS intervention improved the muscle activity and stability of DPN patients during walking. We found that the gait parameters (i.e. gait speed, step length, cadence) of the DPN patients increased after the intervention with RAS. Furthermore, the intervention with RAS led to a moderate decrease in shank muscle co-contraction (measured as CI). Acceleration of the waist also decreased significantly with the RAS intervention with significant improvements observed in all directions except vertical. In contrast, the control group showed mild to moderate improvements in walking speed, step length, and $R M S$ waist acceleration (mediolateral, anteroposterior, 
and total), and there was no significant change in cadence, $C I$, or vertical waist acceleration. The improvements in $C I$ and $R M S$ waist acceleration (mediolateral and total) were significantly greater in the RAS group than in the control group. This remained the case for $C I$ and total acceleration (but not mediolateral acceleration) even adjustment for the covariates.

It has been observed that when participants walk while listening to repetitive auditory stimuli, their gait becomes entrained to the rhythmic signals, resulting in motor unit recruitment patterns with greater consistency. Rhythmic audio patterns increase the excitability of spinal motor neurons via the reticulospinal pathway, reducing the amount of time required for muscles to respond to a given motor command [25]. Muscle activity with the correct timing improves gait stability, as was shown by a previous study using RAS [26]. However, these previous studies did not verify improvements in muscle activity during gait. Our results indicated that RAS intervention resulted in alternation of the rhythmic contractions of the agonist and antagonist muscles.

Our results demonstrated that the addition of walking exercise using RAS improved the gait function of DPN patients when compared to their usual exercise. Previous studies using RAS showed increased gait speed and step length [27], and improved gait rhythm [28], but these studies focused on patients with hemiplegia and Parkinson's disease. To the best of our knowledge, the present study is the first to show the effect of RAS on the gait function of DPN patients. Moreover, we showed this effect with a short-term intervention lasting only two weeks. Previous studies have required a longer period of intervention for effects to become apparent. For example, one program involved resistance exercise and moderate aerobic exercise three times a week for 10 weeks [29], and another involved balanced exercise twice a week for 8 weeks [30]. Fast effectiveness will help in the treatment of patients with deteriorating DPN.

There were a few limitations to this study. The sample size is underpowered to detect changes in gait parameters. In addition, we did not standardize the tempo of the RAS, so exercise intensity may have differed between individuals. Furthermore, we did not standardize the type of footwear; however, because our intention was to measure gait changes in real-world scenarios, we decided to allow the participants to wear prescribed or habitual footwear. In future studies, exercise intensity and gait evaluation circumstances should be standardized.

\section{Conclusion}

The results of this study indicated that the RAS intervention improved gait parameters, co-contraction of the shank muscles, and gait stability in patients with DPN. The improvements in the co-contraction of shank muscles and gait stability were significantly better compared to those with conventional exercise, with this result remaining almost unchanged even after adjustment for covariates. Moreover, the RAS intervention resulted in significantly improved gait function of the DPN patients after only two weeks. Such a rapid effect could be effective 
for and the prevention of falls in patients with DPN.

\section{Acknowledgements}

The authors thank the study participants for their time and dedication. We thank Kazuhiko Mitsutomi, Toshio Utida, Taku Hirooka, Satoshi Konakazawa and Mizuki Watanabe for their contributions to the data collection.

\section{Conflicts of Interest}

The authors declare that there is no conflict of interest associated with this work.

\section{References}

[1] Zochodne, D.W. (2007) Diabetes Mellitus and the Peripheral Nervous System: Manifestations and Mechanisms. Muscle \& Nerve, 36, 144-166. https://doi.org/10.1002/mus.20785

[2] Najafi, B., Khan, T., Fleischer, A. and Wrobel, J. (2013) The Impact of Footwear and Walking Distance on Gait Stability in Diabetic Patients with Peripheral Neuropathy. Journal of the American Podiatric Medical Association, 103, 165-173. https://doi.org/10.7547/1030165

[3] Wrobel, J.S. and Najafi, B. (2010) Diabetic Foot Biomechanics and Gait Dysfunction. Journal of Diabetes Science and Technology, 4, 833-845. https://doi.org/10.1177/193229681000400411

[4] Lin, S., Chen, Y., Liao, C. and Chou, C. (2010) Association between Sensorimotor Function and Forward Reach in Patients with Diabetes. Gait \& Posture, 32, 581-585. https://doi.org/10.1016/j.gaitpost.2010.08.006

[5] Kwon, O., Minor, S., Maluf, K. and Mueller, M. (2003) Comparison of Muscle Activity during Walking in Subjects with and without Diabetic Neuropathy. Gait \& Posture, 18, 105-113. https://doi.org/10.1016/S0966-6362(02)00166-2

[6] Allet, L., Armand, S., Golay, A., Monnin, D., de Bie, R. and de Bruin, E. (2008) Gait Characteristics of Diabetic Patirnts: A Systematic Review. Diabetes Metabolism Research and Reviews, 24, 173-191. https://doi.org/10.1002/dmrr.809

[7] Meier, M.R., Desrosiers, J., Bourassa, P. and Blaszczyk (2001) Effect of Type II Diabetic Peripheral Neuropathy on Gait Termination in the Elderly. Diabetologia, 44, 585-592. https://doi.org/10.1007/s001250051664

[8] Sacco, I.C., Hamamoto, A.N., Onodera, A.N., Gomes, A.A., Weiderpass, H.A., Pachi, C.G., Yamamoto, J.F. and von Tscharner, V. (2014) Motor Strategy Patterns Study of Diabetic Neuropathic Individuals While Walking. A Wavelet Approach. Journal of Biomechanics, 47, 2475-2482. https://doi.org/10.1016/j.jbiomech.2014.04.007

[9] Allet, L., Armand, S., de Bie, R.A., Pataky, Z., Aminian, K., Herrmann, F.R. and de Bruin, E.D. (2009) Gait Alterations of Diabetic Patients While Walking on Different Surfaces. Gait \& Posture, 29, 488-493. https://doi.org/10.1016/j.gaitpost.2008.11.012

[10] Allet, L., Armand, S., de Bie, R.A., Golay, A., Monnin, D., Aminian, K., Staal, J.B. and de Bruin, E.D. (2010) The Gait and Balance of Patients with Diabetes Can Be Improved: A Randomised Controlled Trial. Diabetologia, 53, 458-466. https://doi.org/10.1007/s00125-009-1592-4

[11] Sartor, C.D., Watari, R., Pássaro, A.C., Picon, A.P., Hasue, R.H. and Sacco, I.C. (2012) Effects of a Combined Strengthening, Stretching and Functional Training 
Program versus Usual-Care on Gait Biomechanics and Foot Function for Diabetic Neuropathy: A Randomized Controlled Trial. BMC Musculoskeletal Disorders, 13, 36. https://doi.org/10.1186/1471-2474-13-36

[12] Ahn, S. and Song, R. (2012) Effects of Tai Chi Exercise on Glucose Control, Neuropathy Scores, Balance, and Quality of Life in Patients with Type 2 Diabetes and Neuropathy. The Journal of Alternative and Complementary Medicine, 18, 1172-1178. https://doi.org/10.1089/acm.2011.0690

[13] Ashoori, A., Eagleman, D.M. and Jankovic, J. (2015) Effects of Auditory Rhythm and Music on Gait Disturbances in Parkinson's Disease. Frontiers in Neurology, 6, 234. https://doi.org/10.3389/fneur.2015.00234

[14] Pelton, T.A., Johannsen, L., Chen, H. and Wing, A.M. (2010) Hemiparetic Stepping to the Beat: Asymmetric Response to Metronome Phase Shift during Treadmill Gait. Neurorehabilitation and Neural Repair, 24, 428-434. https://doi.org/10.1177/1545968309353608

[15] Larsson, M. (2014) Self-Generated Sounds of Locomotion and Ventilation and the Evolution of Human Rhythmic Abilities. Animal Cognition, 17, 1-14.

https://doi.org/10.1007/s10071-013-0678-Z

[16] Song, G.B. and Ryu, H.J. (2016) Effects of Gait Training with Rhythmic Auditory Stimulation on Gait Ability in Stroke Patients. Journal of Physical Therapy Science, 28, 1403-1406. https://doi.org/10.1589/jpts.28.1403

[17] Chen, J.L., Penhune, V.B. and Zatorre, R.J. (2008) Listening to Musical Rhythms Recruits Motor Regions of the Brain. Cerebral Cortex, 18, 2844-2854.

https://doi.org/10.1093/cercor/bhn042

[18] Thaut, M.H., McIntosh, G.C. and Hoemberg, V. (2015) Neurobiological Foundations of Neurologic Music Therapy: Rhythmic Entrainment and the Motor System. Frontiers in Psychology, 5, 1185. https://doi.org/10.3389/fpsyg.2014.01185

[19] Yasuda, H., Sanada, M., Kitada, K., Terashima, T., Kim, H., Sakaue, Y., Fujitani, M., Kawai, H., Maeda, K. and Kashiwagi, A. (2007) Kashiwagi, Rationale and Usefulness of Newly Devised Abbreviated Diagnostic Criteria and Staging for Diabetic Polyneuropathy. Diabetes Research and Clinical Practice, 1, 178-183. https://doi.org/10.1016/j.diabres.2007.01.053

[20] Marques, N., Laroche, D., Hallal, C., Crozara, L., Morcelli, M., Karuka, A. and Navega, M. (2013) Gonçalves, Association between Energy Cost of Walking, Muscle Activation, and Biomechanical Parameters in Older Female Fallers and Non-Fallers. Clinical Biomechanics, 28, 330-336. https://doi.org/10.1016/j.clinbiomech.2013.01.004

[21] Falconer, K. and Winter, D.A. (1985) Quantitative Assessment of Co-Contraction at the Ankle Joint in Walking. Electromyogr Clinical Neurophysiology, 25, 135-149.

[22] Sekine, M., Tamura, T., Yoshida, M., Suda, Y., Kimura, Y., Miyoshi, H., Kijima, Y., Higashi, Y. and Fujimoto, T. (2013) A Gait Abnormality Measure Based on Root Mean Square of Trunk Acceleration. Journal of Neuroengineering and Rehabilitation, 10, 118. https://doi.org/10.1186/1743-0003-10-118

[23] Menz, H.B., Lord, S.R. and Fitzpatrick, R.C. (2003) Fitzpatrick, Acceleration Patterns of the Head and Pelvis When Walking on Level and Irregular Surfaces. Gait \& Posture, 18, 35-46. https://doi.org/10.1016/S0966-6362(02)00159-5

[24] DuBois, C.M., Millstein, R.A., Celano, C.M., Wexler, D.J. and Huffman, J.C. (2016) Feasibility and Acceptability of a Positive Psychological Intervention for Patients with Type 2 Diabetes. The Primary Care Companion for CNS Disorders, 18. https://doi.org/10.4088/PCC.15m01902 
[25] Suteerawattananon, M., Morris, G.S., Etnyre, B.R., Jankovic, J. and Protas, E.J. (2004) Effects of Visual and Auditory Cues on Gait in Individuals with Parkinson's Disease. Journal of the Neurological Sciences, 219, 63-69. https://doi.org/10.1016/j.jns.2003.12.007

[26] Sejdić, E., Fu, Y., Pak, A., Fairley, J.A. and Chau, T. (2012) The Effects of Rhythmic Sensory Cues on the Temporal Dynamics of Human Gait. PLOS ONE, 7, e43104. https://doi.org/10.1371/journal.pone.0043104

[27] Park, J., Park, S.Y., Kim, Y.W. and Woo, Y. (2015) Comparison between Treadmill Training with Rhythmic Auditory Stimulation and Ground Walking with Rhythmic Auditory Stimulation on Gait Ability in Chronic Stroke Patients: A Pilot Study. NeuroRehabilitation, 37, 193-202. https://doi.org/10.3233/NRE-151252

[28] Muto, T., Herzberger, B., Hermsdoerfer, J., Miyake, Y. and Poeppel, E. (2012) Poeppel, Interactive Cueing with Walk-Mate for Hemiparetic Stroke Rehabilitation. Journal of NeuroEngineering and Rehabilitation, 9, 58.

https://doi.org/10.1186/1743-0003-9-58

[29] Kluding, P.M., Pasnoor, M., Singh, R., Jernigan, S., Farmer, K., Rucker, J., Sharma, N.K. and Wright, D.E. (2012) The Effect of Exercise on Neuropathic Symptoms, Nerve Function, and Cutaneous Innervation in People with Diabetic Peripheral Neuropathy. Journal of Diabetes and Its Complications, 26, 424-429. https://doi.org/10.1016/j.jdiacomp.2012.05.007

[30] Song, C.H., Petrofsky, J.S., Lee, S.W., Lee, K.J. and Yim, J.E. (2011) Effects of an Exercise Program on Balance and Trunk Proprioception in Older Adults with Diabetic Neuropathies. Diabetes Technology \& Therapeutics, 13, 803-811. https://doi.org/10.1089/dia.2011.0036 